University of Zurich

Department of Economics

Working Paper Series

ISSN 1664-7041 (print)

ISSN 1664-705X (online)

Working Paper No. 187

\title{
On the origin of $r$-concavity and related concepts
}

Tamás L. Balogh and Christian Ewerhart

February 2015 


\title{
On the origin of $r$-concavity and related concepts*
}

\author{
Tamás L. Balogh ${ }^{\dagger}$ \\ Christian Ewerhart ${ }^{\ddagger}$
}

February 25, 2015

\begin{abstract}
In a less widely known contribution, Béla Martos (1966, Hungarian Academy of Sciences) introduced a generalized notion of concavity that is closely related to what is nowadays known as $r$-concavity in the operations research literature, and that is identical to what is nowadays known as $\rho$-concavity in the economics literature. The present paper aims at making the original contribution accessible to a wider audience and illustrating its importance from a modern perspective. To this end, we offer a translation of those parts of Martos (1966) that are directly related to generalized concavity. Reviewing the virtues of $r$-concavity and $\rho$-concavity, we find a surprisingly short proof of the univariate Prékopa-Borell theorem. We also survey a number of applications of the considered concepts in operations research and economics.
\end{abstract}

Keywords: Generalized concavity, $r$-Concavity, $\rho$-Concavity, Nonlinear optimization, Economic applications

\footnotetext{
* This paper was drafted while the first-named author was visiting the University of Zurich as a doctoral student in the academic year 2012/2013. Financial support through a Swiss Government Scholarship (ESKAS 2012.0305) is gratefully acknowledged. We are indebted to the Hungarian Academy of Sciences, especially to Prof. Károly Fazekas, for encouraging us to pursue this research, and for kindly granting us the permission to reproduce Figures 1 and 2 below. Last but not least, we thank Hajnal Máthé for providing valuable assistance in the translation of the Hungarian original.

${ }^{\dagger}$ Department of Economics and Business Administration, University of Debrecen. E-mail: tamas.balogh@econ.unideb.hu, Postal address: Böszörményi út 138, H-4032 Debrecen, Hungary

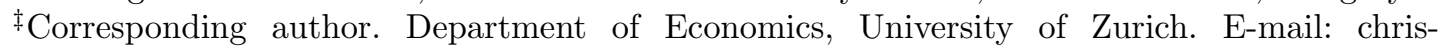
tian.ewerhart@econ.uzh.ch, Postal address: Schönberggasse 1, CH-8001 Zurich, Switzerland
} 


\section{Introduction}

This paper has been written in the honor of Béla Martos who sadly passed away in 2007, leaving a huge scientific heritage. Needless to say, Martos was an authority in the field of operations research. In particular, he made a large number of contributions to the theory of non-linear (e.g., hyperbolic) programming, many of which found useful practical applications. Not all of his contributions, however, are easily accessible for the interested reader.

This remark applies, in particular, to Martos (1966). In that paper, Martos was concerned with exploring the scope of non-linear programming methods. In particular, he defined the concept of $\omega$-concavity, which is closely related to the notion of $r$-concavity that has, by now, been widely used in the operations research literature. In addition, $\omega$-concavity is nothing but $\rho$-concavity as it is used nowadays in the economics literature. Thus, with that paper, Martos conceptualized a main building block of the theory of generalized concavity already in the mid-sixties of the last century.

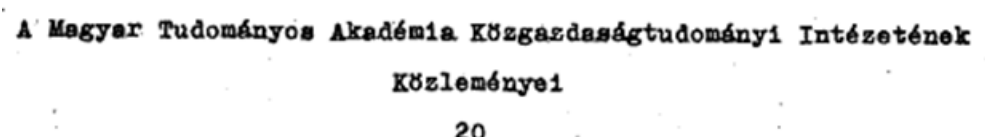

20

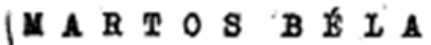 \\ IEM-LINBARIS PROGRAVOZAST MODSZERBK HATOKORB}

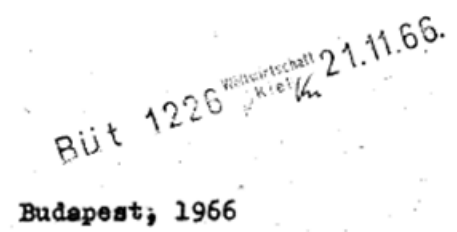

Figure 1: Cover page of Martos (1966); the text has been magnified for better readability

The paper in question, however, was published by the Institute of Economics of the Hungarian Academy of Sciences, and was written almost exclusively in Hungarian. Remarkably, the copy of the paper that we used for the preparation of the 
present article had to be ordered from the archives of the Kiel Institute for the World Economy, Germany. The library stamp imprinted on the cover page (see Figure 1) shows that the paper was already circulated at the year of its appearance, at least at the European scale. However, even in Europe, Hungarian is a language that cannot be easily deciphered by non-natives, due to its unique structure.

The present paper aims at making the original contribution accessible to a wider audience. To this end, we offer a translation of Sections 13 and 14 of Martos (1966) as well as of the relevant parts of his introduction. We review some of the mathematical properties of generalized concavity and offer, in particular, a very short proof of a special case of the Prékopa-Borell theorem. To illustrate the importance of the contribution from a modern perspective, we also survey applications to operations research and economics that have made use of the concept during the last four decades, and especially in recent years.

The remainder of the present paper is structured as follows. Section 2 recalls some definitions. Section 3 reviews early notions of generalized concavity. The impact of Martos (1966) is discussed in Section 4. Section 5 reviews some "virtues" of generalized concavity, including the Prékopa-Borell theorem. Section 6 surveys applications. Translations are provided in Section 7. Section 8 concludes.

\section{The concepts of $r$-concavity and $\rho$-concavity}

The concept of $r$-concavity may be defined as follows (see, e.g., Avriel, 1972, pp. $310-311):^{1}$

Definition 1. A real-valued function $f$ defined on a convex set $C \subseteq \mathbb{R}^{n}$ is said to be $r$-concave, for some $r \in \mathbb{R}$, if

$$
f\left(\lambda x^{1}+(1-\lambda) x^{2}\right) \geq \begin{cases}\frac{1}{r} \ln \left(\lambda e^{r f\left(x^{1}\right)}+(1-\lambda) e^{r f\left(x^{2}\right)}\right) & \text { if } r \neq 0 \\ \lambda f\left(x^{1}\right)+(1-\lambda) f\left(x^{2}\right) & \text { if } r=0\end{cases}
$$

holds for any $x^{1} \in C$, any $x^{2} \in C$, and any $\lambda \in[0,1]$.

\footnotetext{
${ }^{1}$ Some contributions, such as Avriel et al. (2010, p. 240), use a different sign convention. The concept was developed independently by Horst (1971) and Avriel (1972).
} 
In Definition 1, the case $r=0$ may be seen as a limit of the generic case, where $r \rightarrow 0$. Furthermore, the definition extends to infinite values of $r$ in a straightforward way, with $r=-\infty$ corresponding to $f$ being quasiconcave over $C$, and $r=\infty$ corresponding to $f$ being constant over $C$.

A concept closely related to $r$-concavity is what is known as $\rho$-concavity in the economics literature. Caplin and Nalebuff (1991a, p. 29) define $\rho$-concavity as follows: ${ }^{2}$

Definition 2. A real-valued function $g>0$ defined on a convex set $C \subseteq \mathbb{R}^{n}$ is said to be $\rho$-concave, for some $\rho \in \mathbb{R}$, if

$$
g\left(\lambda x^{1}+(1-\lambda) x^{2}\right) \geq \begin{cases}\left(\lambda g^{\rho}\left(x^{1}\right)+(1-\lambda) g^{\rho}\left(x^{2}\right)\right)^{1 / \rho} & \text { if } \rho \neq 0 \\ g^{\lambda}\left(x^{1}\right) g^{1-\lambda}\left(x^{2}\right) & \text { if } \rho=0\end{cases}
$$

holds for any $x^{1} \in C$, any $x^{2} \in C$, and any $\lambda \in[0,1]$.

Also this definition extends to infinite values of the parameter. The close relationship between Definitions 1 and 2 is reflected in the following lemma.

Lemma 1. For any $r=\rho \in[-\infty, \infty]$, a real-valued function $f$ is $r$-concave if and only if $g=e^{f}$ is $\rho$-concave.

Proof. Immediate from the definitions.

Martos (1966) introduced his notion of generalized concavity as $\omega$-concavity, which is defined for both finite and infinite values of the parameter $\omega \in[-\infty, \infty]$. In fact, $\omega$-concavity is identical to $\rho$-concavity when $\omega=\rho$, as can be checked directly by looking at Martos' (1966, Paragraph IV) own English summary of the relevant sections of his paper:

"The transition between the concave and the quasi-concave functions is established by introducing the concept of the $\omega$-concave function. Function $\phi(x)$ being strictly positive for all $x \in X$ is $\omega$-concave in

\footnotetext{
${ }^{2}$ In fact, the definition in Caplin and Nalebuff (1991a) is slightly more general than the one given here in requiring only $g \geq 0$.
} 
set $X$ if for all $x^{1}, x^{2} \in X ; x^{0}=\alpha x^{1}+(1-\alpha) x^{2} ; 0<\alpha<1$ :

$\phi\left(x^{0}\right) \geq\left\{\alpha\left[\phi\left(x^{1}\right)\right]^{\omega}+(1-\alpha)\left[\phi\left(x^{2}\right)\right]^{\omega}\right\}^{1 / \omega}$. For positive functions, this concept leads to the concept of the concave function if $\omega=1$, and to that of the quasi-concave function if $\omega=-\infty$. The flexibility of this generalization points towards a possible direction of further developing the present research. The production functions most widely used in the economic literature are quasi-concave ones (and in the case of increasing returns to scale they are not concave). This allows an economic interpretation of the concept of quasi-concavity and also to realize its economic importance."

Thus, $\omega$-concavity is indeed just what is used these days by economists as $\rho$ concavity. In particular, in view of Lemma 1, the notion of generalized concavity introduced by Martos (1966) corresponds to $r$-concavity via an exponential transformation of the value of the function.

\section{Early notions of generalized concavity}

Research on generalized convex and concave functions has a long tradition in both mathematics and operations research. The influential book by Hardy et al. (1934) introduces generalized means, which appear also in the definition of $r$-concavity. Therefore, one might argue that the definition of generalized concavity is implicit in the definition of a generalized mean. However, this perspective is certainly not the only one possible.

Another early concept of generalized concavity is developed in Beckenbach (1937). His class of super- $F(x)$ functions is defined as follows:

Definition 3. Let $F(x ; \alpha, \beta)$ be a two-parameter family of continuous real-valued functions defined for $a<x<b$ such that for any $a<x_{1}<x_{2}<b$, there is a unique member of the family that takes on given values $y_{1}$ and $y_{2}$ at $x_{1}$ and $x_{2}$, respectively. Then a function $f(x)$ is called super- $F(x, \alpha, \beta)$, or simply super- $F(x)$, 
if $f(x) \geq F_{12}(x)$ for all $x_{1}, x_{2}, x$, with $a<x_{1}<x<x_{2}<b$, where $F_{12}(x)$ denotes the member satisfying $F_{12}\left(x_{1}\right)=f\left(x_{1}\right)$ and $F_{12}\left(x_{2}\right)=f\left(x_{2}\right)$.

A simple relationship between Definitions 1 and 3 is that, if

$$
F(x, \alpha, \beta)=\frac{1}{r} \ln (r(\alpha x+\beta)),
$$

with

$$
\begin{aligned}
& \alpha=\frac{\exp \left(r y_{2}\right)-\exp \left(r y_{1}\right)}{r\left(x_{2}-x_{1}\right)}, \\
& \beta=\frac{x_{2} \exp \left(r y_{1}\right)-x_{1} \exp \left(r y_{2}\right)}{r\left(x_{2}-x_{1}\right)},
\end{aligned}
$$

and $r \in \mathbb{R} \backslash\{0\}$, then the class of super- $F(x)$ functions coincides with the class of functions that are $r$-concave over the interval $(a, b) .{ }^{3}$ While this shows that $r$ concavity is a special case of the $\operatorname{super}-F(x)$ property, there is definitely a lot of value in having also the narrower concept.

Thus, while earlier contributions foreshadowed the specific notions discussed in the present paper, Martos (1966) without doubt accomplished an additional and even necessary step in the development of generalized concavity.

\section{The impact and reception of Martos (1966)}

The following sentence from Section 15 ("Bibliographic remarks") in Martos (1966, p. 81) reveals the author's clear awareness of his contribution:

"To our knowledge, Section 13 contains brand new thoughts. The concepts introduced there - except for the concept of generalized mean-are new, and we hope they will prove to be useful in future research."

As will be seen below (see Section 6), this optimism was not completely without justification. However, as a consequence of the language barrier, subsequent developments did not take an entirely straightforward route. Specifically, in the early

\footnotetext{
${ }^{3}$ To verify this point, one solves the system $F\left(x_{1}, \alpha, \beta\right)=y_{1}, F\left(x_{2}, \alpha, \beta\right)=y_{2}$ for $\alpha$ and $\beta$.
} 
1970s, when research on generalized convexity gained pace, Professor Martos apparently had to remind his colleagues of his ciphertext. For example, at the very end of their survey on quasi-convexity, Greenberg and Pierskalla (1971, pp. 1568-1569) provide a partial account of Martos' contribution, which is - somewhat reluctantlyintroduced as follows:

"In reviewing this paper, Bela Martos kindly pointed out another possibility that yields a continuous-parameter transition from convex to quasiconvex functions. Furthermore, he proved the following theorems. [...]"

Similarly, Avriel (1972, p. 311) writes that he was initially not aware of the closely related work by Martos (1966):

"At the time of writing this article Professor B. Martos brought the attention of the author to the existence of [7], where definition 2.2 (or 2.3) and theorems 3.2, 4.2 and 4.4 are also presented."

Thus, the initial reception of Martos (1966) was not very broad. In his famous monograph on non-linear programming theory, Martos (1975) explicitly refers to his earlier work, but this did not help. In later years, citations of Martos (1966) quickly petered out, and ideas closely related to Martos' original contribution were often attributed to later writings. In particular, with very few exceptions, Martos (1966) is not cited in any of the more recent papers by other authors, except for those written by Hungarian researchers. ${ }^{4}$

\section{$5 \quad$ Virtues of $r$-concavity and $\rho$-concavity}

The widespread use of $r$-concavity and $\rho$-concavity in the literature is owed to a number of useful properties that these concepts possess. In fact, both concepts have their respective merits. For example, $r$-concavity has the advantage of not requiring a positivity constraint, whereas $\rho$-concavity is sometimes more convenient to work

\footnotetext{
${ }^{4}$ Notably, however, Martos (1966) is mentioned by Schaible (1977), Rapcsák and Borzsák (1990), and in the recent monograph on generalized concavity by Avriel et al. (2010).
} 
with. Below, we review three main virtues of these concepts, typically taking $\rho$ concavity as the primitive notion. ${ }^{5}$

A first virtue of the considered notions of generalized concavity is that the property of being $\rho$-concave becomes more stringent as $\rho$ increases. While this ranking property holds generally and may be understood as a consequence of the properties of generalized means, it is instructive at this point to prove the result directly for the special case of a twice continuously differentiable function on the real line.

Lemma 2. [Ranking Property] Let $g>0$ be a twice continuously differentiable $\rho$-concave function on $\mathbb{R}$, where $\rho \in \mathbb{R}$. Then $g$ is also $\rho^{\prime}$-concave for any finite $\rho^{\prime}<\rho$.

Proof. Assume that $\mathrm{g}$ is $\rho$-concave for some $\rho \neq 0$. Then, by definition, $h=g^{\rho} / \rho$ is a concave function. Thus, calculating the second derivative of $h$, one obtains

$$
(\rho-1) g^{\rho-2}\left(g^{\prime}\right)^{2}+g^{\prime \prime} g^{\rho-1} \leq 0 .
$$

Dividing inequality (6) by $g^{\rho-2}>0$, we arrive at

$$
(\rho-1)\left(g^{\prime}\right)^{2}+g^{\prime \prime} g \leq 0
$$

The claim now follows from noting that inequality (7) remains intact when $\rho$ is replaced by a smaller $\rho^{\prime} \neq 0$. The proof is similar if $\rho=0$ or $\rho^{\prime}=0$.

We mention without proof that the ranking result holds also for $\rho^{\prime}=-\infty$, so that in particular, $\rho$-concavity for some finite $\rho \in \mathbb{R}$ implies quasiconcavity.

Another virtue of $r$-concave functions is that they exhibit a predictable behavior under integration, as captured by the Prékopa-Borell theorem. The most general variants of that result relate generalized concavity of densities to generalized concavity of measures on Euclidian spaces. ${ }^{6}$ We state here, again, only a univariate version

\footnotetext{
${ }^{5}$ For a more comprehensive discussion of the mathematical properties of $r$-concave functions, see Zhao et al. (2010).

${ }^{6}$ See the monograph of Dharamadhikari and Joag-dev (1988). Important contributions in this direction were made, in particular, by Prékopa (1971, 1973), Borell (1975), Brascamp and Lieb (1976), Das Gupta (1980), Dancs and Uhrin (1980), and Uhrin (1985, 1994). Also related is Uhrin (1984), who studies the behavior of generalized concave functions under the operation of convolution.
} 
of the theorem for continuously differentiable functions which, however, allows a surprisingly short proof.

Theorem 1. [Prékopa-Borell] Assume that $g>0$ is continuously differentiable and integrable on $\mathbb{R}$, as well as $\rho$-concave for some $\rho>-1$. Then the integral $G(x)=\int_{-\infty}^{x} g(y) d y$ is $\rho^{\prime}$-concave, where $\rho^{\prime}=\rho /(1+\rho)$.

Proof. Suppose that $g$ is $\rho$-concave. Then, $g^{\prime}(x) g^{\rho-1}(x)$ is monotonically decreasing in $x$. Therefore, given that $\rho>-1$,

$$
g^{\prime}(x) g^{\rho-1}(x) G(x)=g^{\prime}(x) g^{\rho-1}(x) \int_{-\infty}^{x} g(y) d y \leq \int_{-\infty}^{x} g^{\prime}(y) g^{\rho}(y) d y=\frac{g^{\rho+1}(x)}{1+\rho} .
$$

Multiplying through with $g^{1-\rho}(x)>0$ yields

$$
g^{\prime}(x) G(x) \leq \frac{g^{2}(x)}{1+\rho},
$$

which is equivalent to $G$ being $\rho^{\prime}$-concave.

A final virtue of the concepts defined above is that they allow a wide range of useful variants. Avriel and Zhang (1974) generalize the original concept by replacing the power transformation with a general transformation of the function value. That approach that has been further extended, in particular, by Ben-Tal (1977), by allowing also for general transformations of the argument. For a specific family of transformations, the possibility of considering such two-sided transformations is mentioned in Avriel (1972). Covering a wider class of generalized concave functions, Chapter 8 of Avriel et al. (2010) provides a useful overview over the theory of concave transformable functions.

\section{Applications}

The above-mentioned virtues of $r$-concavity and $\rho$-concavity might explain why these concepts of generalized concavity could find - and continue to find-many applications in both operations research and economics. ${ }^{7}$

\footnotetext{
${ }^{7}$ The subsequent survey is necessarily incomplete. We apologize to our colleagues for any omissions.
} 


\subsection{Applications to operations research}

There is, of course, a large literature in operations research that makes use of different concepts of generalized concavity. Reflecting the narrower focus of the present paper, we will review selected applications of the discussed concepts to nonlinear programming, stochastic optimization, and integral inequalities.

As for applications to non-linear programming, Avriel (1973) devises an algorithm for minimizing a differentiable convex function over a non-convex set. Specifically, the constraints of the program may be inequalities involving finite sums of generalized convex functions. Interestingly, this analysis was motivated by the economic problem of solving an optimal investment problem under uncertainty. Avriel and Zhang (1974) apply the new concept for deriving an algorithm for a solving certain class of non-linear programs. An early survey on the convexification of nonlinear programs is Horst (1984).

As for applications to stochastic optimization, Rapcsák and Borzsák (1990) investigate how to determine a domain over which the product of several twice continuously differentiable distribution functions is either concave or convex. Dentcheva et al. (2000) consider linear programs in which probabilistic constraints must hold with a given minimum probability. Imposing generalized concavity assumptions on the distributions of integer variables arising in those constraints, the authors derive lower and upper bounds for the optimal value. Zadeh and Khorram (2012) introduce a notion of generalized concavity being related to $r$-concavity, named $h$-concavity. The new concept is used to solve chance-constrained programming problems.

Another direction concerning the operations research applications of $r$-concavity is integral inequalities. Pearce and Pečarić (1996), Gill et al. (1997), and Han and Liu (2012), among others, derive integral inequalities of the Rado and Hadamard types for $r$-convex and $r$-concave functions. Thus, those papers, in essence, derive bounds for an integral using information about the curvature of the integrand and the values of the integrand at the boundary of the integration interval. 


\subsection{Applications to economics}

The application of the above-mentioned concepts of generalized concavity to economic problems occurred only with a certain lag. Moreover, it was $\rho$-concavity that has been used almost exclusively in the economics literature, whereas the term $r$ concavity is probably not known to many economists. The concept of $\rho$-concavity was then employed primarily to measure the curvature of functions with a specific economic meaning, such as demand functions, production functions, and distributions (i.e., density and distribution functions) of individual characteristics. Generalized concavity has been applied, in particular, in the areas of imperfect competition, auctions and mechanism design, public economics, and statistics.

Considering the standard model of imperfect competition, Caplin and Nalebuff (1991a) provide conditions on the distribution of consumer preferences under which a pure-strategy price equilibrium exists. ${ }^{8}$ The curvature of aggregate demand is an important determinant of the impact of price discrimination. Cowan (2007) and Aguirre et al. (2010) offer results on the welfare effects of monopolistic price discrimination. Cowan (2012) investigates the effects of price discrimination on aggregate consumer surplus. Anderson and Renault (2003) apply $\rho$-concavity to the Cournot model to derive efficiency bounds of oligopolistic competition. In another application to the theory of the quantity competition, Ewerhart (2014) uses generalized concavity to derive unifying conditions for existence and uniqueness of a Cournot-Nash equilibrium, the idea being that generalized concavity of the inverse demand function allows to derive upper and lower bounds on the slope of the firms' best-response functions.

Generalized concavity is an assumption that may be imposed on the distribution functions of valuations in auctions and more general mechanisms. An (1998), Bagnoli and Bergström (2005), and Ewerhart (2013) employ generalized concavity to derive,

\footnotetext{
${ }^{8}$ Together with its companion paper discussed below, that paper was first in demonstrating the usefulness of the full-fledged theory of generalized concavity to the economics profession. However, special cases of generalized concavity, such as logconcavity and conditions on the elasticity of functions, obviously have a long tradition in the economics literature. See also the discussion in Dierker (1991).
} 
in particular, sufficient conditions for the important regularity assumption used in mechanism design. Mares and Swinkels (2011) identify a close relationship between $\rho$-concavity of the distribution function and the slope of virtual costs in asymmetric auctions.

A variety of applications exists in public economics. Caplin and Nalebuff (1991b) is a paper in the field of social choice and presents a multi-dimensional mean-voter theorem. Moyes (2003) focuses on how minimal equal-sacrifice taxation can reduce income inequality. He also considers a very general extension of the notion of $\rho$ concavity, allowing not only for an arbitrary monotone transformation of the value of the function but also for another arbitrary monotone transformation of the argument of the function. Also in the realm of public economics, Myatt and Wallace (2005, 2009) deal with so-called collective action games where individual actions have an influence on common consequences. For these games, they determine welfaremaximizing public good production functions.

There are also applications to statistics, a mathematical field that traditionally belongs to economics. For example, Koenker and Mizera (2010) perform maximum likelihood estimation assuming the density is generalized concave. Seregin and Wellner (2010) estimate multivariate densities under the assumption of log-concavity and prove properties of the maximum likelihood estimator for even more general function classes.

\section{Translated sections of Martos' contribution}

This section contains a translation of Sections 13 and 14 of Martos (1966). Together with the previously reviewed Section 15 ("Bibliographic remarks"), these sections form the fourth part "Varia" of Martos (1966). In his introduction, Martos (1966, p. 7) offers the following outlook on that part of the paper:

"In the fourth part, we study different issues that are only loosely connected to each other. In Section 13, we introduce a new, generalized concavity concept and sketch how it could possibly be used in the theory 
of nonlinear programming. Section 14 gives an insight into the economic significance of the mathematical results of the paper. Finally, Section 15 contains bibliographic remarks. In that section, we describe the history of the different concepts and problems, the originality of the results, and their connection to earlier findings."

Here comes the translation of Section 13 (Martos 1966, pp. 70-73). At this point, the reader is informed that, for convenience, we have merged the references used by Martos into our own list of references that can be found at the end of the present article.

\section{"Section 13. A generalization - $\omega$-concave functions}

In this chapter, we report about the preliminary outcome of our research in this field, a research which is still not finished. We hope that, by drawing a link between the concave and quasi-concave function concepts, we will be able to develop the non-linear programming theory towards a higher synthesis. The introduction of the concept of a quasi-concave function into programming theory proved to be fruitful, but we are not able to use many important properties anymore (like, for example, the saddle point theorems, duality, etc.). We do not know yet the outcome of the following generalizations, but we are convinced that it can already help to get a clearer definition of some problems. In this chapter of our study, we do not insist on a totally strict style of argumentation.

Definition 13.1: $\omega$-mean. We define the following as the $\omega$-degree weighted mean (or short: $\omega$-mean) of two positive numbers $\phi_{1}, \phi_{2}$ :

$$
x_{\omega}=x_{\omega}\left(\phi_{1}, \phi_{2}, \alpha\right)=\left[\alpha \phi_{1}^{\omega}+(1-\alpha) \phi_{2}^{\omega}\right]^{1 / \omega} ; \quad 0<\alpha<1 .
$$

The main properties of the $\omega$-mean: ${ }^{9}$

\footnotetext{
${ }^{9}$ Footnote 26 in Martos (1966): "See, e.g., Hardy et al. (1934, p. 13-15 and 26). For more than two numbers the concept can easily be extended."
} 
a If $\phi_{1}=\phi_{2}$ then $x_{\omega}=\phi_{1}$; if $\phi_{1}>\phi_{2}$, then $\phi_{1}>x_{\omega}>\phi_{2}$ for all finite $\omega$.

b If $\omega>\eta$, then $x_{\omega}\left(\phi_{1}, \phi_{2}, \alpha\right) \geq x_{\eta}\left(\phi_{1}, \phi_{2}, \alpha\right)$ and if $\phi_{1} \neq \phi_{2}$ then the inequality is strict.

c Specific $x_{\omega}$-values:

$$
\begin{aligned}
x_{1} & =\alpha \phi_{1}+(1-\alpha) \phi_{2}: \text { the arithmetic mean } \\
x_{-1} & =\frac{\phi_{1} \phi_{2}}{\alpha \phi_{1}+(1-\alpha) \phi_{2}}: \text { the harmonic mean } \\
x_{-\infty} & =\min \left\{\phi_{1}, \phi_{2}\right\} \\
x_{+\infty} & =\max \left\{\phi_{1}, \phi_{2}\right\} \\
x_{0} & =\phi_{1}^{\alpha} \phi_{2}^{1-\alpha}: \text { the geometric mean }
\end{aligned}
$$

We obtain the three last means as limit values.

Definition 13.2: $\omega$-concave function. ${ }^{10}$ Suppose $X \subset E^{n}$ is a convex set, ${ }^{11}$ and suppose $\phi(x)$ is positive on $X .{ }^{12}$ Then $\phi(x)$ on $X$ is a $\omega$-concave function, if for all $x^{1}, x^{2} \in X, x^{0}=\alpha x^{1}+(1-\alpha) x^{2}$ (where $0<\alpha<1$ )

$$
\phi_{0} \geq x_{\omega}\left(\phi_{1}, \phi_{2}, \alpha\right)
$$

where $\phi_{i}=\phi\left(x^{i}\right), i=0,1,2$.

If $1 / \phi(x)$ is $-\omega$-concave then $\phi(x)$ is $\omega$-convex.

According to this definition, we see that 1-concavity corresponds to Gconcavity, whereas $-\infty$-concavity corresponds to G-quasi-concavity. ${ }^{13}$ If $-\infty<\omega<1$, then we will obtain-according to the requirements

\footnotetext{
${ }^{10}$ Footnote 27 in Martos (1966): "We restrict attention to weak $\omega$-concavity. Using this model, it is easy to define strong and strict $\omega$-concavities as well."

${ }^{11}$ Translator's note: $E^{n}$ denotes the $n$-dimensional Euclidean space. Moreover, the set-theoretic inclusion symbol refers to a weak relation, i.e., $X=E^{n}$ is consistent with $X \subset E^{n}$.

${ }^{12}$ Footnote 28 in Martos (1966): "This condition decreases the possibility of generalization quite a lot."

${ }^{13}$ Translator's note: G-concavity in this context refers to weak concavity, whereas G-quasiconcavity refers to weak quasi-concavity. The latter is defined as follows (Martos 1966, p. 100): A scalar-valued vector function $\phi(x)$ is called weakly quasi-concave (G-quasi-concave) in a convex set $X \subset E^{n}$, if $x^{1}, x^{2} \in X, x^{0} \in\left(x^{1}, x^{2}\right)$ implies $\phi\left(x^{0}\right) \geq \min \left\{\phi\left(x^{1}\right), \phi\left(x^{2}\right)\right\}$.
} 
of the concavity - continuously changing versions situated between the above two versions. Furthermore, we see, as a result of the characteristics defined in property $b$ of the $\omega$-mean, that if $\omega>\eta$, then each $\omega$-concave function is $\eta$-concave (in fact, even strongly $\eta$-concave).

Definition 13.3: Positive $\omega$-combination of positive functions. Suppose $\phi(x), \psi(x)$ are functions with a positive value for any $x \in X$, $\lambda_{1}, \lambda_{2}$ are positive numbers, and $\omega$ is a real number. We call the function below a positive $\omega$-combination of the functions $\phi(x)$ and $\psi(x)$ :

$$
\phi_{\omega}(x)=\phi_{\omega}\left[\phi(x), \psi(x), \lambda_{1}, \lambda_{2}\right]=\left\{\lambda_{1}\left[\phi(x)^{\omega}\right]+\lambda_{2}\left[\psi(x)^{\omega}\right]\right\}^{1 / \omega}
$$

In the case $\omega=1$, we obtain the concept of positive linear combination, while for $\omega=0$,

$$
\phi_{0}(x)=[\phi(x)]^{\lambda_{1}}[\psi(x)]^{\lambda_{2}}
$$

Theorem 13.4 The positive $\omega$-combination of $\omega$-concave functions is also $\omega$-concave.

Proof. Using the notations of Definitions 13.1-13.3, suppose that the two functions to combine, $\phi(x)$ and $\psi(x)$, are $\omega$-concave, which means:

$$
\begin{aligned}
\phi_{0} \geq x_{\omega}\left(\phi_{1}, \phi_{2}, \alpha\right) & =\left[\alpha \phi_{1}^{\omega}+(1-\alpha) \phi_{2}^{\omega}\right]^{1 / \omega} \\
\psi_{0} \geq x_{\omega}\left(\psi_{1}, \psi_{2}, \alpha\right) & =\left[\alpha \psi_{1}^{\omega}+(1-\alpha) \psi_{2}^{\omega}\right]^{1 / \omega}
\end{aligned}
$$

Thus,

$$
\begin{aligned}
& \rho_{\omega 0}=\left(\lambda_{1} \phi_{0}^{\omega}+\lambda_{2} \psi_{0}^{\omega}\right)^{1 / \omega} \geq \\
& \geq\left\{\lambda_{1}\left(\alpha \phi_{1}^{\omega}+(1-\alpha) \phi_{2}^{\omega}\right)+\lambda_{2}\left(\alpha \psi_{1}^{\omega}+(1-\alpha) \psi_{2}^{\omega}\right)\right\}^{1 / \omega}= \\
& =\left[\alpha \rho_{\omega 1}^{\omega}+(1-\alpha) \rho_{\omega 2}^{\omega}\right]^{1 / \omega} .
\end{aligned}
$$

This theorem, which is actually a generalization of the well-known theorem of the positive linear combination of concave functions, can be used 
as a basis for our further research. Namely, using this statement for the case $\omega=-\infty$, we get nothing but a weakened version of Proposition 5.18 (applying to positive functions only). ${ }^{14}$ However, we think that, if it can be proved that quasi-concave functions that fulfill certain regularity conditions are $\omega$-concave with a finite $\omega$, then for these functions a stronger combination theorem will be applicable. And, as a result, we might be able to create a generalized Lagrange-function with saddle point property."

After having defined the concept of $\omega$-concavity, Martos outlines a number of economic applications in his Section 14. Here is a translation of that section (Martos 1966, pp. 73-77):

\section{"Section 14: The significance of the results in economics}

The practical economic importance of the mathematic results discussed in chapters 6-7 is obviously the fact that they allow to extend the range of the methods discussed in chapters 9-12 to new types of functions. ${ }^{15}$ However, the following question arises: are there really any economic relations which lead to such types of functions (like for example the quasi-concave functions) and, if yes, how can the mathematical concept be interpreted in the language of economics.

In response to the first question, we may first recall the case of aggregated production functions. In the economics literature, we find almost exclusively the following three types of production functions: ${ }^{16}$

\footnotetext{
${ }^{14}$ Translator's note: Proposition 5.18 (Martos 1966, p. 25) reads as follows: "If a function $\phi(x)$ is G-quasiconcave on a set $\mathrm{X}$, then $\psi(x)=\phi(A x+b)$ is G-quasiconcave on the set $Y=\{x \mid A x+b \in X\} . "$

${ }^{15}$ Translator's note: Chapters 6 and 7 present theorems concerning feasible areas and optimal solutions of non-linear programming exercises. Chapters 9-12 deals with extending of the range of certain methods to solve non-linear programming exercises.

${ }^{16}$ Footnote 29 from Martos (1966): "For the description of the production functions, we will deviate from our previously used notations and prefer the traditional notations instead. Thus: $Q=$ output, $K=$ price of the capital goods, $L=$ labor input (scalar variables). The Greek letters stand for constant values whose economic interpretation is to be found in the literature on production theory. See Frisch (1963) and Schreiner (1964)."
} 
a The linear production function of Harrod (1946) and Domar (1948):

$$
Q=\frac{1}{\chi} K
$$

b The generalized Cobb-Douglas (Cobb and Douglas, 1928) log-linear production function:

$$
Q=\gamma K^{\alpha} L^{\beta}
$$

c The Arrow-Chenery-Minhas-Solow's (ACMS) (Arrow et al., 1961) constant elasticity of substitution production function:

$$
Q=\gamma\left[\delta K^{-\rho}+(1-\delta) L^{-\rho}\right]^{-1 / \rho}
$$

Regarding these functions we can state the following: ${ }^{17}$

a The Harrod-Domar function, as the simplest linear production function, has always been easy to handle.

b As far as the generalized Cobb-Douglas production function is concerned, let us suppose that $L>0, K>0, Q>0$ (which means that $\gamma>0$ ) and that the marginal productivity of both the capital good and labor are positive, i.e., $\alpha>0, \beta>0$. In this case, it is easy to see from the definition that the function is $\frac{1}{\alpha+\beta}$-concave, and so, as a result of $\alpha+\beta>0$, it is 0 -concave, consequently quasiconcave. Furthermore, if $\alpha+\beta \leq 1$ (diminishing/constant returns to scale), then it is 1-concave, that is: concave. But in the case $\alpha+\beta>1$ (increasing returns to scale), the function is not concave. There has barely been any research on the case of increasing returns to scale until now, due to the fact that the non-concavity property made the function very difficult to handle. Nevertheless, the circumstance that the function is, in any case, quasi-concave

\footnotetext{
${ }^{17}$ Footnote 30 in Martos (1966) "We consider those functions in their univariate or bivariate forms. The multivariate generalization of the Cobb-Douglas function is trivial; as for the ACMS, see the works of Uzawa (1962) and Mukerji (1963)."
} 
(in fact, even 0-concave), makes it possible to maximize the output with limited resources, or even to minimize the input costs when output is bounded from below. This is true even if the production function (which is the objective function in the first case, yet the function in the constraint in the second case) is a Cobb-Douglastype function with increasing returns to scale. Nevertheless, we note that the maximization of the net profit $(p Q-r K-w L$, where $p$, $r, w$ are prices) is still unsolved due to the fact the function is not quasi-concave.

c Similar observations can be made about the ACMS functions. If $\gamma>0$ and $0<\delta<1$, then $Q>0$, and if also $\nu>0$, then the differential productivities are also positive. It is easy to see that if, in addition, the assumption $\rho \geq-1$ is satisfied, then the function is $\left(\frac{-\rho}{\nu}\right)$-concave, so it is quasi-concave (it is even strongly quasi-concave), but it is concave only if $\rho \leq-\nu$. In the case of this function, $\nu$ is the degree of homogeneity (just as $\alpha+\beta$ in the case of the Cobb-Douglas function), so $\nu>1$ corresponds to the increasing returns to scale. So in the case of increasing returns to scale, $\rho \geq-1 \geq-\nu$, which means that the ACMS function is not concave. But because it is strongly quasi-concave, the above mentioned analysis is valid for its optimization.

It is obvious to conclude from these examples that the concept of nonconcave but quasi-concave functions has an important role in the theory of "increasing returns to scale"-type production functions.

After all this, we can go back to the second part of the question which we formulated at the beginning of this chapter: how can quasi-concavity be interpreted from an economic point of view? 
Let us consider the twice differentiable production function

$$
Q=\phi(K, L)
$$

which satisfies inequalities $Q>0, \phi_{K}=\frac{\partial \phi}{\partial K}>0, \phi_{L}=\frac{\partial \phi}{\partial L}>0$ over the set $K>0, L>0$. Consider now the curves $\phi(K, L)=Q_{0}$ (level lines, isoquants) on the $(K, L)$ plane which belong to the fixed production levels $Q_{0}$. If now $\phi(K, L)$ is G-quasi-concave on the $K>0, L>0$ set, thenas follows from Proposition $6.3^{18}$ - the set defined by $\phi(K, L) \geq Q_{0}$ is convex, or - in a simplified manner - the isoquants turn their convexity towards the origin. It also follows from Proposition 6.3 that this can only be true for all isoquants if $\phi(K, L)$ is G-quasi-concave (Fig. 6). ${ }^{19}$

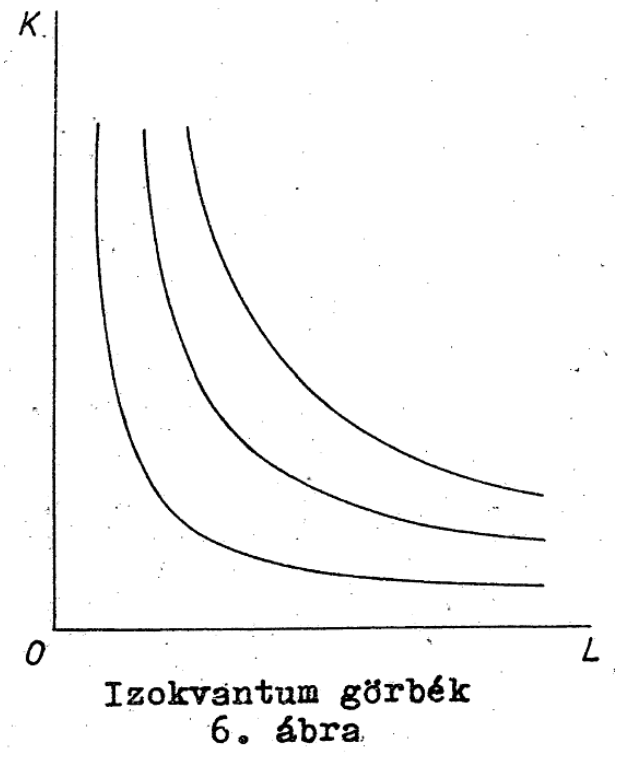

Figure 2: Figure 6 of Martos (1966). The text under the figure means: "Isoquant curves, 6th figure"

Along a given isoquant, capital goods and work input are substitutes.

The proportion of these two values changes along the curve, so we may

\footnotetext{
${ }^{18}$ Translator's note: Proposition 6.3 (Martos 1966, p. 30) reads as follows: "Let $X$ be a convex set. The set $L(\beta)=\{x \mid \gamma(x) \leq \beta, x \in X\}$ is convex for all $\beta$ if and only if $\gamma(x)$ is G-quasiconvex on $X$."

${ }^{19}$ Translator's note: Figure 6 from page 76 of the original article is reproduced as Figure 2 in the present paper.
} 
only speak about a marginal rate of substitution. From the differentiation rule for implicit functions, we obtain the marginal rate of substitution as follows:

$$
\frac{d K}{d L}=-\frac{\phi_{L}}{\phi_{K}}
$$

This is the slope of the tangent in point $(K, L)$ to the isoquant crossing the point $(K, L)$. We assume that $\phi_{L}>0, \phi_{K}>0$, which means that the substitution rate is negative. This is actually quite clear: the decrease in labor is substituted by an increase in capital goods and vice versa.

Let us analyze how this substitution rate changes if we move along the fixed curve further in the direction of an increasing $L$ :

$$
\frac{d^{2} K}{d L^{2}}=\frac{1}{\phi_{L}^{3}}\left(2 \phi_{K} \phi_{L} \phi_{K L}-\phi_{K}^{2} \phi_{L L}-\phi_{L}^{2} \phi_{K K}\right)=\frac{1}{\phi_{L}^{3}}\left|\begin{array}{ccc}
0 & \phi_{K} & \phi_{L} \\
\phi_{K} & \phi_{K K} & \phi_{K L} \\
\phi_{L} & \phi_{L K} & \phi_{L L}
\end{array}\right|
$$

The last formula contains a bordered Hessian determinant. We assume that $1 / \phi_{L}^{3}$ is positive, so that the sign depends on the sign of the determinant. Arrow and Enthoven (1961) have proved that if the function $\phi(K, L)$ is quasi-concave, then the determinant is non-negative (Arrow et al. 1961). Furthermore, reversing the theorem (partially) we can state: if the determinant is positive then the function is G-quasi-concave. This roughly means that a quasi-concave production function corresponds to a negative and increasing substitution rate (in absolute values a decreasing substitution rate) and vice versa. In other words, the quasi-concave production function means: the less developed the technology used to sustain a certain production level, the less capital goods are needed to substitute one unit of labor. This assumption sounds quite rational and gives a satisfactory explanation for the important role of quasi-concave functions."

The remainder of Martos (1966) summarizes the state of nonlinear optimization techniques at the time and will, therefore, not be reviewed herein. 


\section{Conclusion}

The first variant of what is nowadays known as $r$-concavity was introduced by Béla Martos (1966). In later work, Avriel (1972), in particular, rediscovered the concept, offering an extended definition and proving a wider range of results. Due to its simplicity and useful mathematical properties, this comparably narrow concept of generalized concavity has led to numerous useful applications in both operations research and economics. We believe that this concept deserves even more attention and is likely to see further fruitful development in the future.

\section{References}

[1] Aguirre I, Cowan S, Vickers J (2010) Monopoly price discrimination and demand curvature, American Economic Review, 100, 1601-1615

[2] An MY (1998) Logconcavity versus logconvexity: A complete characterization, Journal of Economic Theory, 80, 350-369

[3] Anderson SP, Renault R (2003) Efficiency and surplus bounds in Cournot competition, Journal of Economic Theory, 113, 253-264

[4] Arrow KJ, Chenery HB, Minhas BS, Solow RM (1961) Capital-labor substitution and economic efficiency, Review of Economics and Statistics, 43, 225-248

[5] Arrow KJ, Enthoven AC (1961) Quasi-concave programming, Econometrica, 29, $779-800$

[6] Avriel M (1972) r-conxex functions, Mathematical Programming, 2, 309-323

[7] Avriel M (1973) Solution of certain nonlinear programs involving r-convex functions, Journal of Optimization Theory and Applications, 11, 159-174

[8] Avriel M, Zang I (1974) Generalized convex functions with applications to nonlinear programming, in: Moeseke PV (ed.), Mathematical Programs for Activity Analysis, North Holland, New York, USA, 23-33 
[9] Avriel M, Diewert VE, Schaible S, Zang I (2010) Generalized concavity. Classics in Applied Mathematics No. 63, Society for Industrial and Applied Mathematics, Philadelphia, USA

[10] Bagnoli M, Bergström T (2005) Log-concave probability and its applications, Economic Theory, 26, 445-469

[11] Beckenbach EF (1937) Generalized convex functions, Bulletin of the American Mathematical Society, 43, 363-371

[12] Ben-Tal A (1977), On generalized means and generalized convex functions, Journal of Optimization Theory and Applications, 21, 1-13

[13] Borell C (1975) Convex Set Functions in d-Space, Periodica Mathematica Hungarica, 6, 111-136

[14] Brascamp HJ, Lieb EH (1976) On the extensions of the Brunn-Minkowski and Prékopa-Leindler theorems, including inequalities for log-concave functions, and with an application to the diffusion equation, Journal of Functional Analysis, 22, 366-389

[15] Caplin A, Nalebuff B (1991a) Aggregation and imperfect competition: on the existence of equilibrium, Econometrica, 59, 25-59

[16] Caplin A, Nalebuff B (1991b) Aggregation and social choice: a mean voter theorem, Econometrica, 59, 1-23

[17] Cobb CW, Douglas PH (1928) A theory of production, American Economic Review, 18, 139-165

[18] Cowan S (2007) The welfare effects of third-degree price discrimination with nonlinear demand functions, The RAND Journal of Economics 38, 419-428

[19] Cowan S (2012) Third-degree price discrimination and consumer surplus, Journal of Industrial Economics, 60, 333-345 
[20] Dancs S, Uhrin B (1980) On a class of integral inequalities and their measuretheoretic consequences, Journal of Mathematical Analysis and Applications, 74, $388-400$

[21] Das Gupta S (1980) Brunn-Minkowski inequality and its aftermath, Journal of Multivariate Analysis, 10, 296-318

[22] Dentcheva D, Prékopa A, Ruszczynski A (2000) Concavity and efficient points of discrete distributions in probabilistic programming, Mathematical programming, $89,55-77$

[23] Dharmadhikari S, Joag-dev K (1988) Unimodality, convexity, and applications, Academic Press, Boston.

[24] Dierker E (1991) Competition for consumers, in: Barnett WA (ed.) Equilibrium theory and applications: proceedings of the Sixth International Syposium in Economic Theory and Econometrics, Cambridge University Press, Cambridge, 383-402

[25] Domar ED (1946) Capital expansion, rate of growth and employment, Econometrica, 14, 137-147

[26] Ewerhart C (2013) Regular type distributions in mechanism design and $\rho$ concavity, Economic Theory, 53, 591-603

[27] Ewerhart C (2014) Cournot games with biconcave demand, Games and Economic Behavior, 85, 37-47

[28] Frisch R (1963) Lois techniques et economiques de la production, Dunod, Paris

[29] Gill PM, Pearce CEM, Pečarić (1997) Hadamard's inequality for $r$-convex functions, Journal of Mathematical Analysis and Applications, 215, 461-470

[30] Greenberg HJ, Pierskalla WP (1971) A review of quasi-convex functions, Operations Research, 19, 1553-1570 
[31] Han LX, Liu GF (2012) Integral inequalities of Hermite-Hadamard type for r-convex functions, Applied Mathematics, 3, 1967-1971

[32] Hardy GH, Littlewood JE, Pólya G (1934) Inequalities, Cambridge University Press, Cambridge

[33] Harrod RF (1948) Towards a Dynamic Economics, Macmillan, London

[34] Horst R (1971) Über mittelbar konvexe Optimierung, PhD thesis, Technical University of Damstadt

[35] Horst R (1984) On the convexification of non-linear programming problems: An applications-oriented survey, European Journal of Operations Research, 15, $382-392$

[36] Koenker R, Mizera I (2010) Quasi-concave density estimation, Annals of Statistics, 38, 2998-3027

[37] Mares V, Swinkels JM (2011) Near-optimality of second price mechanisms in a class of asymmetric auctions, Games and Economic Behavior, 72, 218-241

[38] Martos B (1966) Nem-lineáris programozási módszerek hatóköre, A Magyar Tudományos Akadémia Közgazdaságtudományi Intézetének Közleményei, MTA, Budapest.

[39] Martos B (1975) Nonlinear Programming Theory and Methods, North-Holland, New York

[40] Moyes P (2003) Redistributive effects of minimal equal sacrifice taxation, Journal of Economic Theory, 108, 111-140

[41] Mukerji V (1963), A generalized SMAC function with constant ratios of elasticities of substitution, Review of Economic Studies, 30, 233-236

[42] Myatt DP, Wallace C (2005) Production targets and free disposal in the private provision of public goods, University of Oxford Economics Discussion Paper 231. 
[43] Myatt DP, Wallace C (2009) Evolution, teamwork and collective action: Production targets in the private provision of public goods, Economic Journal, 119, $61-90$

[44] Pearce CEM, Pečarić J (1996) A continuous analogue and an extension of Radó's formulate for convex and concave functions, Bulletin of the Australian Mathematical Society, 53, 229-233

[45] Prékopa A (1971) Logarithmic concave measures with applications to stochastic programming, Acta Scientiarum Mathematicarum (Szeged), 32, 301-315

[46] Prékopa A (1973) On logarithmic concave measures and functions, Eötvös Loránd University, Budapest, Hungary

[47] Rapcsák T, Borzsák P (1990) On separable product functions, Optimization, $21,831-841$

[48] Schaible S (1977) Second-order characterizations of pseudoconvex quadratic functions, Journal of Optimization Theory and Applications, 21, 15-26

[49] Schreiner E (1964) Produktionstheorie, Handworterbuch der Sozialwissenschaften Bd. 8., G. Fischer, Stuttgart

[50] Seregin A, Wellner JA (2010) Nonparametric estimation of multivariate convextransformed densities, Annals of Statistics, 38 (6), 3751-3781

[51] Uhrin B (1984) Some remarks about the convolution of unimodal functions, Annals of Probability, 12, 640-645

[52] Uhrin B (1985) Extensions and sharpenings of Brunn-Minkowski and Bonnesen inequalities, Colloquia Mathematica Societatis János Bolyai 48 Intuitive Geometry, Siófok, Hungary, 551-571

[53] Uhrin B (1994) Curvilinear extensions of the Brunn-Minkowski-Lustenik inequality, Advances in Mathematics, 109, 288-312 
[54] Uzawa H (1962) Production functions with constant elasticities of substitution, Review of Economic Studies, 29, 291-299

[55] Zadeh ZM, Khorram E (2012) Convexity of chance constrained programming problems with respect to a new generalized concavity notion, Annals of Operations Research, 196, 651-662

[56] Zhao YX, Wang SY, Coladas Uria L (2010) Characterizations of $r$-convex functions, Journal of Optimization Theory and Applications, 145, 186-195 